\title{
The change of coagulation profile in two- staged arthroplasty for periprosthetic joint infection patients: a retrospective cohort study
}

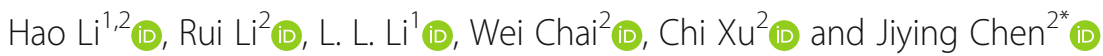

\begin{abstract}
Aims: Periprosthetic joint infection (PJI) is a serious complication of total joint arthroplasty. We performed a retrospective cohort study to evaluate (1) the change of coagulation profile in two-staged arthroplasty patients and (2) the relationship between coagulation profile and the outcomes of reimplantation.

Method: Between January 2011 and December 2018, a total of 202 PJI patients who were operated on with twostaged arthroplasty were included in this study initially. This study continued for 2 years and the corresponding medical records were scrutinized to establish the diagnosis of PJI based on the 2014 MSIS criteria. The coagulation profile was recorded at two designed points, (1) preresection and (2) preimplantation. The difference of coagulation profile between preresection and preimplantation was evaluated. Receiver operating characteristic curves (ROC) were used to evaluate the diagnostic efficiency of the coagulation profile and change of coagulation profile for predicting persistent infection before reimplantation.
\end{abstract}

Results: The levels of APT, INR, platelet count, PT, T, and plasma fibrinogen before spacer implantation were significantly higher than before reimplantation. No significant difference was detected in the levels of D-dimer, ACT, and AT3 between the two groups. The AUC of the combined coagulation profile and the change of combined coagulation profile for predicting persistent infection before reimplantation was $0.667(95 \% \mathrm{Cl} 0.511,0.823)$ and 0.667 (95\% Cl 0.526, 0.808), respectively.

Conclusion: The coagulation profile before preresection is different from before preimplantation in two-staged arthroplasty and the coagulation markers may play a role in predicting infection eradication before reimplantation when two-stage arthroplasty is performed.

Level of evidence: Level III, diagnostic study.

Keywords: Periprosthetic joint infection, Total joint arthroplasty, Two-staged arthroplasty, Coagulation profile, Activated partial thromboplastin time

\footnotetext{
* Correspondence: chenjiyingpla@sina.com

${ }^{2}$ Department of Orthopedic Surgery, The First Medical Center, Chinese PLA General Hospital, 28 Fuxing Road, Beijing, People's Republic of China

Full list of author information is available at the end of the article
}

(c) The Author(s). 2021 Open Access This article is licensed under a Creative Commons Attribution 4.0 International License, which permits use, sharing, adaptation, distribution and reproduction in any medium or format, as long as you give appropriate credit to the original author(s) and the source, provide a link to the Creative Commons licence, and indicate if changes were made. The images or other third party material in this article are included in the article's Creative Commons licence, unless indicated otherwise in a credit line to the material. If material is not included in the article's Creative Commons licence and your intended use is not permitted by statutory regulation or exceeds the permitted use, you will need to obtain permission directly from the copyright holder. To view a copy of this licence, visit http://creativecommons.org/licenses/by/4.0/ The Creative Commons Public Domain Dedication waiver (http://creativecommons.org/publicdomain/zero/1.0/) applies to the data made available in this article, unless otherwise stated in a credit line to the data. 


\section{Introduction}

Total joint arthroplasty (TJA) has been one of the most successful surgeries during the last century. Patients with advanced joint diseases can achieve relief of pain and functional recovery after this surgery. However, periprosthetic joint infection (PJI) is a disastrous complication after total joint arthroplasty and often indicates unfavorable outcomes [1,2]. Although two-staged arthroplasty is a preferred treatment for chronic PJI, it is still difficult to predict persistent infection before reimplantation [3] and inappropriate implantations can lead to treatment failure. A failure of two-stage reimplantation can result in more disastrous complications and the need for further arthroplasty, arthrodesis, and/or amputation [4].

The pathogens of PJI are known to impair their hosts by endotoxin and exotoxin which can stimulate immune cells to produce various cytokines such as IL-6, TNF, and IL-6 [5-7]. These secreted cytokines may disrupt normal coagulation cascade and cause abnormal coagulation profiles in PJI patients. Some studies revealed that the coagulation profile of PJI patients was different from that of non-PJI patients. It suggests that PJI patients suffer from abnormal coagulation. Theoretically, the subclinical abnormal coagulation can increase the risk of epidural hematoma formation and impair incision healing after surgery [6-8]. However, a review of the literatures suggests that the studies about the relationship between the coagulation system and the clinical outcomes of PJI are limited [9-12]. We hold the opinion that the effects of pathogens on the coagulation system may disappear, and the corresponding abnormal coagulation profile may return to normal when the pathogens are eradicated because the endotoxin and exotoxin produced by pathogens are removed.

Based on what was mentioned, we propose a hypothesis that the coagulation profile of PJI patients before reimplantation is different from before spacer implantation when the infection was controlled and the changed coagulation profile can play a role in predicting persistent infection before reimplantation. Some studies revealed that plasma fibrinogen can play a role in predicting persistent infection before reimplantation in two-stage exchange arthroplasty for PJI. However, there is still a lack of comprehensive studies evaluating the change of coagulation profiles in two-staged exchange arthroplasty and the association between the coagulation profile before reimplantation, and the outcomes of two-stage exchange arthroplasty $[13,14]$.

In a bid to address the problems mentioned above, we performed a retrospective cohort study with at least 2 years of follow-up to evaluate (1) the change of the coagulation profile in two-staged arthroplasty PJI patients and (2) the use of coagulation profile in predicting persistent infection before reimplantation.

\section{Materials and methods}

\section{Inclusion and exclusion criteria}

Institutional review board approval was obtained prior to the commencement of this study, and then, a total of 202 PJI patients treated with two-staged arthroplasty are included in this study. All of the patients were performed with two-staged arthroplasty for PJI treatment in our center. The inclusion criteria were (1) patients diagnosed with PJI by 2014 MSIS criteria and (2) PJI patients treated with 2-staged arthroplasty.

The exclusion criteria were (1) patients who were exposed to anticoagulation agents within 2 weeks before reimplantation and preresection; (2) periprosthetic fracture; (3) periprosthetic dislocation; (4) patients who received coronary stents, filter implantation, and internal fixation implantation; (5) patients who did not receive new prosthesis reimplantation after spacer implantation; and (6) patients who receive spacer implantation in other joint centers. A total of 130 patients were included in this study; the details are shown in Fig. 1. The medical records of PJI patients were scrutinized and the diagnosis of PJI was based on the 2014 MSIS criteria.

Furthermore, the following data was collected to reflect the coagulation profile of PJI patients: APTT, TT, INR, prothrombin time $(\mathrm{PT})$, plasma $\mathrm{D}$-dimer, plasma fibrinogen, plasma $\mathrm{Ca}+$, platelet count, antithrombin 3, and prothrombin activity (PTA).

\section{Treatment protocol}

According to the institutional standard of two-stage arthroplasty, the two-stage arthroplasty is performed by several surgeons. During the first stage of two-stage arthroplasty, all implanted prostheses are removed, then followed by an extensive debridement and irrigation; betadine and hydrogen peroxide is used separately for the irrigation. Irrigate the infected joint three times accompanied by pulsed washes with saline. Then, an articular antibiotic-loaded cement spacer (polymethylmethacrylate, PMMA) is inserted. The antibiotics added to the bone cement are selected based on the preoperative culture result and antibiotic sensitivity test. In the case of culture-negative PJI, an articular antibiotic-loaded cement spacer containing $8 \mathrm{~g}$ vancomycin and $4 \mathrm{~g}$ meropenem per $40 \mathrm{~g}$ bone cement is then implanted.

After the spacer implantation, 6 weeks of IV antibiotics is administrated based on tests of antibiotic sensitivity, followed by 6 weeks of po. antibiotics until the infection is controlled. An antibiotic holiday of at least 2 weeks is stipulated before reimplantation.

The timing of reimplantation was based on the following criteria:

1) No clinical symptoms and signs of joint infection such as fistula, pain, and effusions 


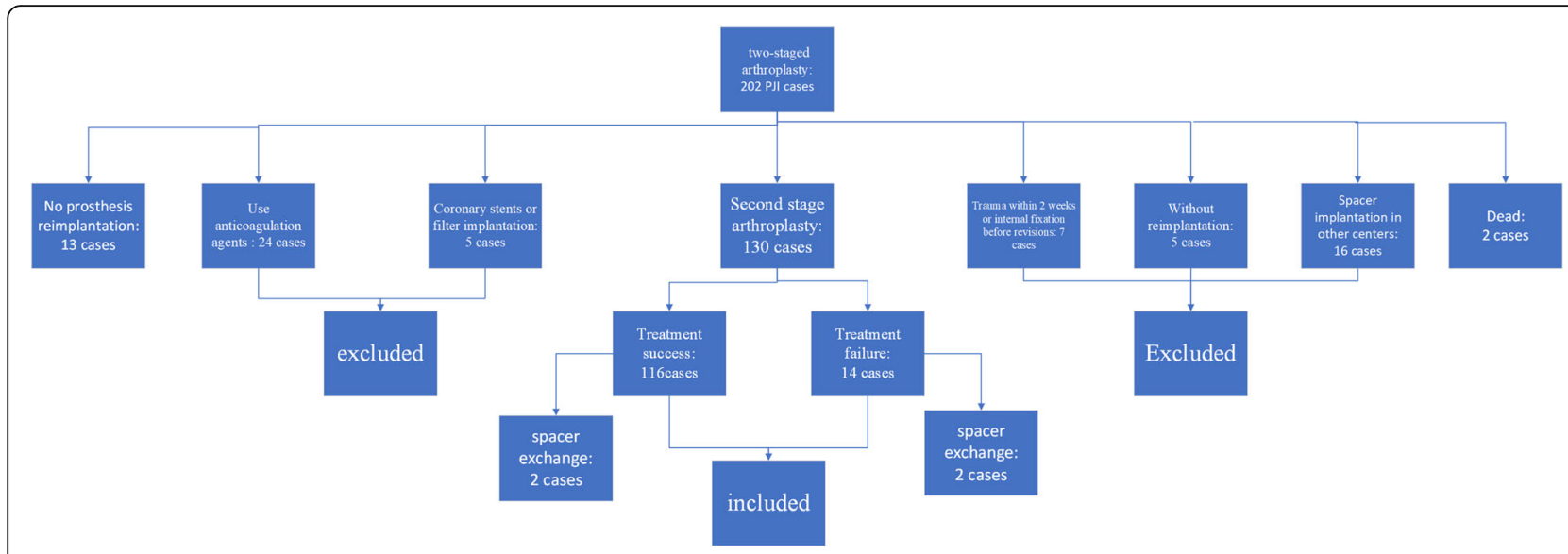

Fig. 1 Patients' details

2) A gradual decrease in plasma ESR and CRP after discontinuation of antibiotic administration

\section{Definition of treatment failure and treatment success}

All PJI patients were checked up upon for 2 years after reimplantation and the outcomes of follow-up were used to evaluate the prognosis of 2-staged reimplantation. Patients were checked on at 3 months, 6 months, 12 months, and 24 months after surgery. Follow-up visits include outpatient and remote follow-up visits. Imaging data (radiographs and pictures of the healing of the postoperative incision) for remote follow-up visits were taken locally and the imaging data was collected in the form of electronic photos. During the follow-up period after reimplantation, if PJI was suspected, a joint aspiration and subsequent synovial fluid analysis was performed.

Treatment failure was defined as:

1) Re-infection after reimplantation based on the MSIS criteria

2) Two identical pathogens revealed by at least two intraoperative periprosthetic cultures at reimplantation

The Delphi-based criteria were used to define treatment success; they were summarized as:

1) A healed incision without fistula, pain, effusions, and recurrence of infection caused by the same pathogen.

2) No surgery was performed on the infected joint after reimplantation.

3) No mortality due to PJI.

\section{Statistical analysis}

The variables were divided into continuous variables and dichotomous data based on the types of data. A normal distribution test was used to evaluate the distribution of continuous variables. The continuous variables were described as means if the normal distribution was achieved. Otherwise, corresponding medians were calculated. Rank sum test and Student t-test were used to detect the difference if the corresponding applicable conditions were met. Dichotomous data were described as frequencies and compared by chi-squared test subsequently. $\mathrm{P}<0.05$ indicates statistical significance. Paired tests were used to compare the difference of coagulation profile between preresection and preimplantation. Receiver operating characteristic curves (ROC) were used to evaluate the efficiency of the coagulation profile in predicting persistent infection before reimplantation. Logistic regression was used to build diagnostic models based on the coagulation profile. Yonder's index was used to identify optimal cut-off. SPSS (IBM; version 26.0) was used to perform statistical analysis.

\section{Results}

\section{Demographic characteristics}

The median ages in the treatment success group and the treatment failure group were 63 and 60 years, respectively. The median BMI in the success group and failure groups were $23.95 \mathrm{~kg} / \mathrm{m}^{2}$ and $28.72 \mathrm{~kg} / \mathrm{m}^{2}$, respectively. The percentage of females in the success group and failure group was $58.62 \%$ and $35.71 \%$, respectively. The percentage of the knee in the success group and failure group was $42.24 \%$ and $57.14 \%$, respectively. The percentage of patients with inflammatory joint diseases in the success group and failure group was $5.17 \%$ and $7.14 \%$, respectively. The details of the demographic characteristics are summarized in Table 1.

\section{The change of coagulation profile in PJI two-staged arthroplasty}

The levels of APTT, INR, platelet count, PT, and plasma fibrinogen before spacer implantation (group A) were 
Table 1 Demographic characteristics

\begin{tabular}{|c|c|c|c|}
\hline & $\begin{array}{l}\text { Treatment } \\
\text { success } \\
N=116\end{array}$ & $\begin{array}{l}\text { Treatment } \\
\text { failure } \\
n=14\end{array}$ & $P$ values \\
\hline Age & $63(27,84)$ & $60(24,76)$ & 0.942 \\
\hline Female ( $n, \%)$ & $68,58.62 \%$ & $5,35.71 \%$ & 0.103 \\
\hline Knee $(n, \%)$ & $49,42.24 \%$ & $8,57.14 \%$ & 0.288 \\
\hline$B M I^{*}$ & $\begin{array}{l}23.95(16.6, \\
36.49)\end{array}$ & $\begin{array}{l}28.72(22.1 \\
28.7)\end{array}$ & 0.108 \\
\hline \multicolumn{4}{|l|}{$A S A$} \\
\hline 2 & $63,54.31 \%$ & $10,71.42 \%$ & 0.223 \\
\hline 3 & $6,5.17 \%$ & 0 & 1 \\
\hline 4 & 0 & 0 & 1 \\
\hline Liver disease( $n, \%)$ & $3,2.59 \%$ & 0 & 1 \\
\hline Kidney disease $(n, \%)$ & $1,0.86 \%$ & 0 & 1 \\
\hline Heart disease $(n, \%)$ & $9,7.76 \%$ & $1,7.14 \%$ & 1 \\
\hline$D M(n, \%)$ & $19,16.38 \%$ & 0 & 1 \\
\hline $\begin{array}{l}\text { Inflammatory joint } \\
\text { diseases }(n, \%)\end{array}$ & $6,5.17 \%$ & $1,7.14 \%$ & 1 \\
\hline Spacer interval(day)* & $120.5(32,535)$ & $144(52,308)$ & 0.47 \\
\hline \multicolumn{4}{|l|}{ Causative pathogens } \\
\hline CNS & $50,43.10 \%$ & $2,14.29 \%$ & 0.038 \\
\hline $\begin{array}{l}\text { Staphylococcus } \\
\text { aureus }\end{array}$ & $7,6.03 \%$ & $4,28.57 \%$ & 0.019 \\
\hline Enterococcus spp. & $5,4.31 \%$ & 0 & 1 \\
\hline Streptococcus spp. & $8,6.90 \%$ & $1,7.14 \%$ & 1 \\
\hline $\begin{array}{l}\text { Gram-negative } \\
\text { bacteria }\end{array}$ & $10,8.62 \%$ & $4,28.57 \%$ & 0.045 \\
\hline Fungi & $5,4.31 \%$ & 0 & 1 \\
\hline Other pathogens & $6,5.26 \%$ & $1,7.14 \%$ & 0.559 \\
\hline Culture negative & $40,34.48 \%$ & $3,21.43 \%$ & 0.385 \\
\hline
\end{tabular}

*Values were given as medians (minimum, maximum)

significantly higher than before reimplantation (group B). No significant difference was detected in the levels of plasma D-dimer and AT3 between the two groups. The median APTT in group A was 38.3s and 35.9s in group B. The median PT in group A was $13.5 \mathrm{~s}$ and $13.3 \mathrm{~s}$ in group B. The median TT in group A was $15.9 \mathrm{~s}$ and $15.85 \mathrm{~s}$ in group $\mathrm{B}$. The median INR in group A was 1.04 and 1.02 in group B. The median plasma fibrinogen in group A was $4.86 \mathrm{~g} / \mathrm{L}$ and $3.36 \mathrm{~g} / \mathrm{L}$ in group B. The median platelet count in group A was 277109/dl and 195 $109 / \mathrm{dl}$ in group $\mathrm{B}$. The median plasma $\mathrm{Ca}$ in group A was $2.23 \mathrm{mmol} / \mathrm{L}$ and $2.26 \mathrm{mmol} / \mathrm{L}$ in group $\mathrm{B}$. The details about the change of coagulation profiles are shown in Table 2. No significant difference was revealed between the coagulation profile in the treatment success group than that of in the treatment failure group. The details of the coagulation profile before reimplantation (treatment failure versus treatment success) are shown in Table 3.
Table 2 The change of coagulation profile in PJI two-staged arthroplasty

\begin{tabular}{llll}
\hline Coagulation profile & Preresection & Preimplantation & P values \\
\hline APTT(s) & $38.3(18.1,62.9)$ & $35.9(23.7,51.6)$ & $<0.001^{*}$ \\
PT(s) & $13.5(11.7,39)$ & $13.3(11.9,16.8)$ & $0.016^{*}$ \\
TT(s) & $15.9(14,21.1)$ & $15.85(4,19.1)$ & 0.183 \\
INR & $1.04(0.86,1.97)$ & $1.02(0.87,1.38)$ & $0.003^{*}$ \\
Plasma fibrinogen (g/L)** & $4.86(2.30,8.08)$ & $3.36(1.21,8.9)$ & $<0.001^{*}$ \\
Plasma Ca (mmol/L) & $2.23(1.2,2.27)$ & $2.26(1.9,2.58)$ & 0.158 \\
AT3 (\%) & $86(2.28,125)$ & $89(2.88,129)$ & 0.306 \\
D-dimer (ug/ml) & $1.57(0.2,6.39)$ & $1.49(0.17,12.27)$ & 0.577 \\
Platelet count (10\%/dl) & $277(262,292)$ & $207(196,218)$ & $<0.001^{*}$ \\
\hline${ }^{*} P<0.05$ & & \\
Values were given as medians (minimum, maximum) & \\
${ }^{*}$ values were given as means $(95 \% \mathrm{Cl})$ & &
\end{tabular}

The association between the coagulation profile and treatment outcomes of two-staged arthroplasty

The diagnostic value of the coagulation profile was evaluated in ROC curves (Fig. 2). The AUCs of plasma D-dimer, platelet count, APTT, TT, and INR before reimplantation for predicting persistent infection were $0.542(0.359,0.725), 0.560(0.417,0.702), 0.526(0.377$, $0.674), 0.482(0.29,0.674)$, and $0.632(0.479,0.786)$, respectively. Furthermore, the coagulation profile was combined by logistic regression (Additional file 1: Appendix 1.) based on significantly upregulated markers. The AUC of the combined coagulation profile was 0.667 (95\% CI $0.511,0.823)$. The diagnostic efficiency of the coagulation profile before re-implantation is depicted in Table 4. Besides, the diagnostic values of the change in coagulation profile from pre-resection to pre-implantation

Table 3 The difference of coagulation profile between controlled infection group and persistent infection group before reimplantation

\begin{tabular}{llll}
\hline $\begin{array}{l}\text { Coagulation } \\
\text { profile }\end{array}$ & Treatment success & Treatment failure & $P$ value \\
\hline $\boldsymbol{A P T T ^ { a }}$ & $35.3(23.7,46.1)$ & $37.05(33.8,45.5)$ & 0.755 \\
$\boldsymbol{P T}$ & $13.4(11.9,16.8)$ & $13.2(12.3,15.1)$ & 0.188 \\
$\boldsymbol{T} \boldsymbol{T}$ & $15.8(4,18.6)$ & $15.1(13.5,16.8)$ & 0.827 \\
INR & $1.02(0.89,1.38)$ & $1.01(0.93,1.19)$ & 0.106 \\
Plasma fibrinogen & $3.19(1.21,6.1)$ & $3.88(3.12,6.44)$ & 0.822 \\
Plasma Ca & $2.22(1.9,2.51)$ & $2.22(2.01,2.39)$ & 0.583 \\
AT3 & $91(2.88,129)$ & $90(77,116)$ & 0.552 \\
D-dimer & $1.49(0.17,11.68)$ & $1.1(0.69,4.72)$ & 0.668 \\
Platelet count & $196.23(175.95$, & $195(152.48$, & 0.468 \\
& $216.51)$ & $237.52)$ & \\
ESR & $12(2,36)$ & $11(4,30)$ & 0.706 \\
CRP & $0.298(0.05,4.46)$ & $0.71(0.1,2.57)$ & 0.220 \\
\hline
\end{tabular}

Values were given as medians (minimum, maximum)

a Values were given as means $(95 \% \mathrm{Cl})$ 




Fig. 2 The diagnostic value of the coagulation profile evaluated in ROC curves

Table 4 The association between the coagulation profile and treatment outcomes of two-staged arthroplasty. Corresponding cutoff and predictive values

\begin{tabular}{|c|c|c|c|c|c|}
\hline & $A \cup C(95 \% \mathrm{Cl})$ & Yonden index & Optimal cut-off & Sensitivity & specificity \\
\hline \multicolumn{6}{|c|}{ The coagulation profile before reimplantation } \\
\hline APTT & $0.526(0.377,0.674)$ & 0.170 & 33.45 & 92.9 & 24.1 \\
\hline$P T$ & $0.608(0.454,0.762)$ & 0.3 & 13.15 & 58.6 & 28.6 \\
\hline$T T$ & $0.482(0.29,0.674)$ & 0.158 & 16.05 & 57.1 & 41.4 \\
\hline D-dimer & $0.542(0.359,0.725)$ & 0.262 & 1.19 & 66.2 & 60 \\
\hline Platelet count & $0.560(0.417,0.702)$ & 0.188 & 231.5 & 33 & 85.7 \\
\hline INR & $0.632(0.479,0.786)$ & 0.335 & 1.005 & 62.1 & 71.4 \\
\hline ESR & $0.465(0.282,0.647)$ & 0.083 & 31 & 8.3 & 100 \\
\hline$C R P$ & $0.615(0.427,0.803)$ & 0.318 & 0.33 & 81.8 & 50 \\
\hline Combined coagulation profile & $0.667(0.511,0.823)$ & NA & NA & NA & NA \\
\hline \multicolumn{6}{|c|}{ The change of coagulation profile from preresection to preimplantation } \\
\hline APTT & $0.546(0.378,0.714)$ & 0.145 & -3.8 & 78.6 & 21.4 \\
\hline PT & $0.545(0.392,0.699)$ & 0.175 & 0.95 & 18.3 & 81.7 \\
\hline$\pi$ & $0.493(0.327,0.659)$ & 0.096 & 2.5 & 10 & 100 \\
\hline D-dimer & $0.611(0.403,0.819)$ & 0.278 & -0.395 & 70.7 & 57.1 \\
\hline Platelet count & $0.528(0.374,0.682)$ & 0.248 & 56.5 & 63.3 & 61.5 \\
\hline INR & $0.626(0.488,0.765)$ & 0.235 & 0.05 & 57.1 & 66.4 \\
\hline Plasma fibrinogen & $0.549(0.363,0.736)$ & 0.227 & 0.365 & 79.8 & 57.1 \\
\hline Combined coagulation profile & $0.667(0.526,0.808)$ & NA & NA & NA & NA \\
\hline
\end{tabular}


were shown in ROC curves (Fig. 3). The AUCs of $\triangle \mathrm{D}$ dimer, $\Delta$ plasma fibrinogen, $\Delta$ platelet count, $\Delta$ APTT, $\Delta \mathrm{PT}$, $\triangle \mathrm{INR}$, and $\triangle \mathrm{TT}$ for infection eradication were 0.611 (0.403, 0.819), 0.549 (0.363, 0.736), 0.528 (0.374, 0.682), 0.546 (0.378, 0.714), 0.545 (0.392, 0.699$), 0.626(0.488$, $0.765)$, and $0.493(0.327,0.659)$, respectively. The AUC of the combined coagulation profile was $0.667(0.526,0.808)$. The diagnostic efficiency of the change in coagulation profile from pre-resection to pre-implantation is shown in Table 4. Besides, the change of coagulation profile was combined by logistic regression (Additional file 2: Appendix2).

\section{Discussion}

There is still a lack of comprehensive studies that evaluate the change of coagulation profile from reresection to re-implantation in two-staged arthroplasty up to now $[3,15,16]$. To address this problem, this study evaluated the change in coagulation profile during this period and the use of coagulation profile for predicting infection eradication in two-staged arthroplasty.

PJI pathogens can secret endotoxins and exotoxins by which stimulate immune cells to produce cytokines such IL-6, TNF- $\alpha$, and IL-6 [5, 6]. These cytokines can impair the normal coagulation system subsequently. Parvizi et al. revealed that the INR in PJI patients is higher than that in aseptic loosening patients [6]. These studies indicated that PJI patients suffered from abnormal hypocoagulation. Our previous studies have also shown similar results. The changed coagulation cascade may be ascribed to PJI pathogens and return to relatively normal when the pathogens were eradicated. This research revealed that the levels of APTT, PT, INR, plasma fibrinogen, and platelet count before reimplantation were significantly lower than that of before re-resection. Unfortunately, no significant difference was detected when these variables were compared between the infection-controlled and persistent infection groups. This may be the result of a small number of persistent infection patients.

The goal of two-staged arthroplasty for PJI treatment is to eradicate infection. However, defining the time for re-implantation is still challenging and previous studies failed to identify serologic markers to guide the time for reimplantation $[3,16]$. Therefore, we evaluate the efficiency of the coagulation profile for predicting persistent infection. We found that the levels of INR and APTT before re-implantation may predict infection eradication despite relatively poor efficiency. Moreover, we built a logistic model based on the change of INR, APTT, and platelet count. This model performed better than anyone alone and some commonly used serologic markers such as ESR and CRP in predicting persistent infection.

The levels of APTT, INR, and platelet count before reimplantation were significantly lower than those before spacer implantation. Therefore, we evaluated whether the change of these coagulation markers $(\triangle \mathrm{APTT}, \Delta \mathrm{INR}$, and $\Delta$ platelet count) from pre-resection to pre-implantation

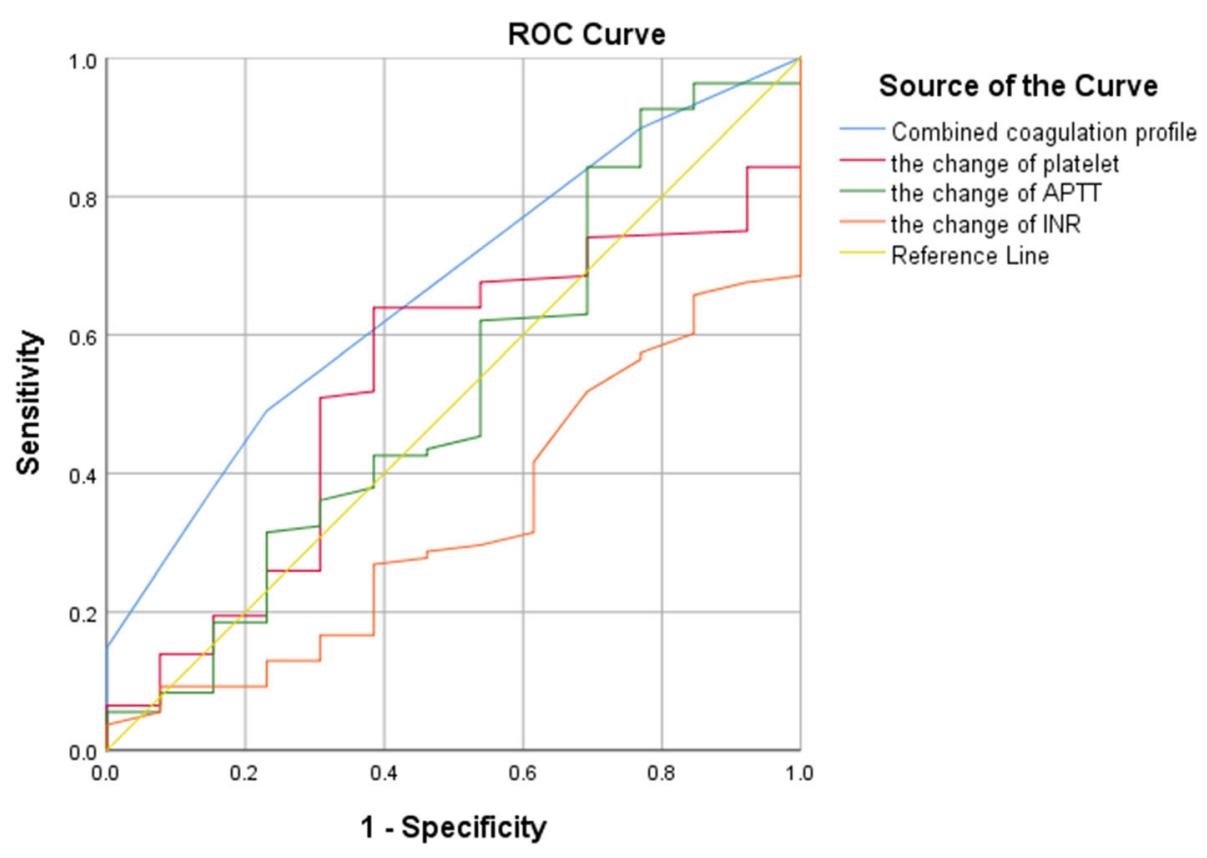

Diagonal segments are produced by ties.

Fig. 3 The diagnostic values of the change in coagulation profile from pre-resection to pre-implantation shown in ROC curves 
could indicate infection eradication. The change of coagulation profile may help doctors guide the timing of re-implantation. In 14 recurrent PJI cases, 7 cases underwent elevated APTT, INR, and platelet count $(\triangle \mathrm{APTT}>0$, $\triangle \mathrm{INR}>0$, and $\Delta$ platelet count $>0$ ). However, no statistical significance was detected between the recurrent rate in the elevated group $(\triangle \mathrm{APTT}>0, \Delta \mathrm{INR}>0$ and $\Delta$ platelet count $>0)$ and that of in the non-elevated group $(\triangle \mathrm{APTT}<$ $0, \Delta \mathrm{INR}<0$ and $\Delta$ platelet count $<0$ ) because the sample size of recurrent infection is relatively small.

Other studies used modified MSIS criteria or repeat cultures as the indicator of persistent infection $[17,18]$. However, these methods were not used in this study because repeat cultures were fraught with high false negative rates, and modified MSIS criteria were of limited efficiency. Besides, no good criteria are accepted commonly as the standard of persistent infection before re-implantation. Therefore, we hold the opinion that the follow-up outcomes of PJI patients can be a relatively accurate indicator for infection eradication because many PJI criteria are built based on the follow-up results [18]. The relatively long follow-up period is one of our advantages over other studies.

A literature review suggested that many researchers failed to find accurate serological markers that indicated persistent infection such as ESR and CRP, and our results revealed similar results. Some synovial fluid indicators can predict persistent infection such as synovial WBC count and the percentage of PMN $[3,19]$. These findings indicated that the local response to persistent infection was more accurate than the systemic response to the diagnosis of persistent infection. However, this study revealed that the combination of coagulation profiles was of comparable efficiency compared to that of reported synovial fluid indicators.

D-dimmer is a product of fibrin degradation and fibrinogen is the precursor of fibrin. But the fluctuation in plasma fibrinogen and D-dimer was not detected in the same direction. Then we reviewed the original medical data and found that many data about the levels of plasma D-dimer were not accessible because this test was not performed regularly in our center. Therefore, the statistical power is not strong enough to detect the difference in the levels of plasma D-dimer between pre-resection and pre-implantation because of the relatively small sample size compared to that of plasma fibrinogen.

The levels of APTT and INR before pre-resection is higher than those before re-implantation. It suggests that intrinsic and extrinsic coagulation pathways changed before re-implantation compared to before pre-resection. Relatively robust TT revealed that the common pathway in the coagulation cascade did not change significantly between pre-resection and pre-implantation. However,

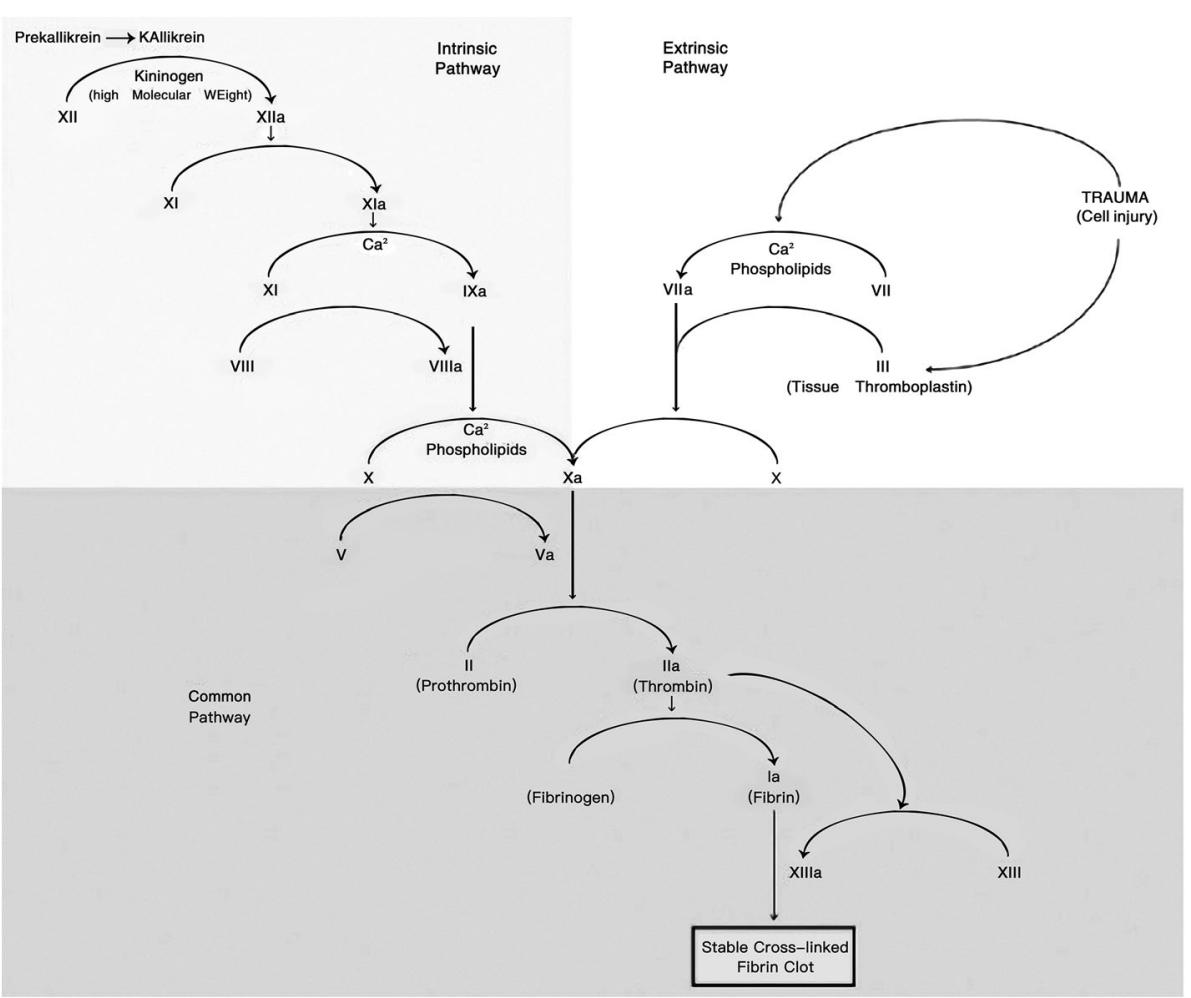

Fig. 4 Part of intrinsic and extrinsic coagulation pathways' changes 
as shown in Fig. 4 [6], part of intrinsic and extrinsic coagulation pathways' changes significantly was not explored in this study. For example, which changed coagulation factor in intrinsic and extrinsic pathway cause the change of APTT and INR remains unknown. Moreover, this field needs further exploration.

We have shown that the coagulation profile may play a role in guiding the timing of implantation. The decrease in the levels of APTT and INR may indicate infection eradication and surgeons can take these markers into account before re-implantation. We believe that surgeons should continue to combine various diagnostic tools rather than a certain single marker to guide the timing of re-implantation, including the change of coagulation profile, serologic markers, clinical characteristics, and so on. For example, reduced APTT, INR, and platelet count before re-implantation compared to before pre-section may predict a higher success rate of re-implantation. How to identify the timing of reimplantation still needs to be further explored.

This study still has some limitations. First, two-staged arthroplasty is a relatively successful surgery and the rate of treatment failure is low in our center. Therefore, a large sample size is needed to reach statistical significance when comparing the difference of coagulation profile between the successful group and the persistent infection group. However, the sample size in this study is limited after strict inclusion and exclusion. Second, this study was performed in a tertiary center. The patients admitted to our joint center often suffered from severe infection and received previous treatments in other joint centers so that spacer exchange was not rare in this tertiary center. This fact can trigger a selection bias. Third, there is no gold standard for the diagnosis of infection eradication and we use follow-up results to evaluate infection eradication. However, some subclinical infections may be missed by patients or doctors during follow-up. This fact can trigger some biases. Finally, some patients underwent repeat debridement and spacer exchange before re-implantation. The decision of this was based on a combination of clinical appearance and laboratory tests. Besides, the patients who refused to receive re-implantation after spacer implantation and the patients who receive spacer implantation in other joint centers were excluded from this study. These conditions can also add bias to this study.

\section{Conclusion}

The coagulation profile is different between pre-resection and pre-implantation in two-staged arthroplasty; the coagulation markers may play a role in predicting infection eradication before re-implantation when two-stage arthroplasty is performed.

\section{Abbreviations}

TJA: Total joint arthroplasty; PJl: Periprosthetic joint infection;

MSIS: Musculoskeletal Infection Society criteria; PT: Prothrombin time;

APTT: Activated partial thromboplastin time; INR: International normalized

ratio; PTA: Prothrombin activity; TT: Thrombin time

\section{Supplementary Information}

The online version contains supplementary material available at https://doi. org/10.1186/s13018-021-02477-4.

Additional file 1: Appendix 1.

Additional file 2: Appendix 2.

\section{Acknowledgements}

This article is mainly completed by Dr. Hao Li. Jiying Chen and Libao Hao are the correspondent authors and guide the writing of this article. Rui Li, LL Li, Wei Chai, and Chi Xu contribute to the data collection. Jun Fu and Libo Hao provided valuable comments during revisions.

\section{Authors' contributions}

Study design: Hao Li and Rui Li. Data collection/validation: Hao Li and LL Li. Data analysis: Hao Li. Result interpretation: Hao Li. Reporting and editing: Rui $\mathrm{Li}$ and Chi Xu. Final approval of the version to be submitted: Jiying Chen and Wei Chai. Project guarantor: Jiying Chen and Wei Chai. The authors read and approved the final manuscript.

\section{Authors' information}

Hao Li, Rui Li, LL Li, Wei Chai, Chi Xu, LiBao Hao, and Jiying Chen come from the Department of Orthopedics, Chinese People's Liberation Army General Hospital (301 Hospital), 28 Fuxing Rd., 100853, Beijing, PR China.

\section{Funding}

National Key Research and Development Program of China (No.2020YFC2004900)

Availability of data and materials

All data and materials were in full compliance with the journal's policy.

\section{Declarations}

\section{Ethics approval and consent to participate}

This study was approved by the institutional review board of our hospital (Chinese People's Liberation Army General Hospital). This study was performed in accordance with the ethical standards in the 1964 Declaration of Helsinki.

Consent for publication

We have obtained consent to publish from the participants.

Competing interests

All authors declare that they have no competing interests.

\section{Author details}

${ }^{1}$ Medical School of Chinese PLA, Beijing, People's Republic of China.

${ }^{2}$ Department of Orthopedic Surgery, The First Medical Center, Chinese PLA

General Hospital, 28 Fuxing Road, Beijing, People's Republic of China.

Received: 22 January 2021 Accepted: 11 May 2021

Published online: 18 May 2021

References

1. Kapadia BH, Berg RA, Daley JA, Fritz J, Bhave A, Mont MA. Periprosthetic joint infection. Lancet. 2016;387(10016):386-94.

2. Wainwright TW, Gill M, McDonald DA, Middleton RG, Reed M, Sahota O, et al. Consensus statement for perioperative care in total hip replacement and total knee replacement surgery: Enhanced Recovery After Surgery (ERAS( $\left.{ }^{\oplus}\right)$ ) Society recommendations. Acta Orthop. 2020;91(1):3-19. https:// doi.org/10.1080/17453674.2019.1683790. 
3. Kusuma SK, Ward J, Jacofsky M, Sporer SM, Della Valle CJ. What is the role of serological testing between stages of two-stage reconstruction of the infected prosthetic knee? Clini Orthop Relat Res. 2011;469(4):1002-8. https:// doi.org/10.1007/s11999-010-1619-7.

4. Sayeed Z, Anoushiravani AA, El-Othmani MM, Chambers MC, Mihalko WM, Jiranek WA, et al. Two-stage revision total knee arthroplasty in the setting of periprosthetic knee infection. Instr Course Lect. 2017;66:249-62.

5. Levi M, Keller TT, van Gorp E, ten Cate H. Infection and inflammation and the coagulation system. Cardiovas Res. 2003;60(1):26-39. https://doi.org/10.1 016/50008-6363(02)00857-X.

6. Saxena A, Baratz M, Austin MS, Purtill JJ, Parvizi J. Periprosthetic joint infection can cause abnormal systemic coagulation. J Arthroplasty. 2011; 26(1):50-7, 7.e1

7. Tapper $\mathrm{H}$, Herwald H. Modulation of hemostatic mechanisms in bacterial infectious diseases. Blood. 2000;96(7):2329-37. https://doi.org/10.1182/blood. V96.7.2329.

8. Cancienne JM, Werner BC, Browne JA. Complications after TKA in patients with hemophilia or Von Willebrand's disease. J Arthroplasty. 2015;30(12): 2285-9. https://doi.org/10.1016/j.arth.2015.06.015.

9. Nelson CL, Jones RB, Wingert NC, Foltzer M, Bowen TR. Sonication of antibiotic spacers predicts failure during two-stage revision for prosthetic knee and hip infections. Clin Orthop Relat Res. 2014;472(7):2208-14. https:// doi.org/10.1007/s11999-014-3571-4.

10. Shahi A, Kheir MM, Tarabichi M, Hosseinzadeh HRS, Tan TL, Parvizi J. Serum D-dimer test is promising for the diagnosis of periprosthetic joint infection and timing of reimplantation. J Bone Joint Surg Am. 2017;99(17):1419-27. https://doi.org/10.2106/JBJS.16.01395.

11. Xu H, Xie J, Huang Q, Lei Y, Zhang S, Pei F. Plasma fibrin degradation product and D-dimer are of limited value for diagnosing periprosthetic joint infection. J Arthroplasty. 2019;34(10):2454-60. https://doi.org/10.1016/j.arth.2 019.05.009.

12. Li R, Shao HY, Hao LB, Yu BZ, Qu PF, Zhou YX, et al. Plasma fibrinogen exhibits better performance than plasma D-dimer in the diagnosis of periprosthetic joint infection: a multicenter retrospective study. J Bone Joint Surg Ame. 2019:101(7):613-9. https://doi.org/10.2106/JBJS.18.00624.

13. Wu H, Meng Z, Pan L, Liu H, Yang X, Yongping C. Plasma fibrinogen performs better than plasma d-dimer and fibrin degradation product in the diagnosis of periprosthetic joint infection and determination of reimplantation timing. J Arthroplasty. 2020;35(8):2230-6. https://doi.org/10.1 016/j.arth.2020.03.055.

14. Pannu TS, Villa JM, Riesgo AM, Patel PD, Barsoum WK, Higuera-Rueda CA. Serum D-dimer in the diagnosis of periprosthetic knee infection: where are we today? J Knee Surg. 2020;33(2):106-10. https://doi.org/1 $0.1055 / \mathrm{s}-0039-1698467$.

15. Ghanem E, Azzam K, Seeley M, Joshi A, Parvizi J. Staged revision for knee arthroplasty infection: what is the role of serologic tests before reimplantation? Clin Orthop Relat Res. 2009;467(7):1699-705. https://doi. org/10.1007/s11999-009-0742-9.

16. Bin $G$, Xinxin $Y$, Fan $L$, Shenghong $W$, Yayi $X$. Serum fibrinogen test performs well for the diagnosis of periprosthetic joint infection. J Arthroplasty. 2020;35(9):2607-12. https://doi.org/10.1016/j.arth.2020.04.081.

17. Xu H, Xie J, Yang J, Chen G, Huang Q, Pei F. Plasma fibrinogen and platelet count are referable tools for diagnosing periprosthetic joint infection: a single-center retrospective cohort study. J Arthroplasty. 2020;35(5):1361-7. https://doi.org/10.1016/j.arth.2019.12.015.

18. Parvizi J, Tan TL, Goswami K, Higuera C, Della Valle C, Chen AF, et al. The 2018 Definition of periprosthetic hip and knee infection: an evidence-based and validated criteria. J Arthroplasty. 2018;33(5):1309-14.e2.

19. Shukla SK, Ward JP, Jacofsky MC, Sporer SM, Paprosky WG, Della Valle C Perioperative testing for persistent sepsis following resection arthroplasty of the hip for periprosthetic infection. J Arthroplasty. 2010;25(6 Suppl):87-91. https://doi.org/10.1016/j.arth.2010.05.006.

\section{Publisher's Note}

Springer Nature remains neutral with regard to jurisdictional claims in published maps and institutional affiliations.

\section{Ready to submit your research? Choose BMC and benefit from:}

- fast, convenient online submission

- thorough peer review by experienced researchers in your field

- rapid publication on acceptance

- support for research data, including large and complex data types

- gold Open Access which fosters wider collaboration and increased citations

- maximum visibility for your research: over $100 \mathrm{M}$ website views per year

At BMC, research is always in progress.

Learn more biomedcentral.com/submissions 